\title{
UN ENFOQUE METODOLÓGICO PARA EL MODELAMIENTO ESPACIAL DE SERVICIOS AMBIENTALES EN EL SUR DE CHILE
}

Gerardo Vergara Asenjo ${ }^{1}$

\section{RESUMEN}

Los servicios ambientales de los bosques han adquirido una creciente importancia, generando beneficios directos $\mathrm{e}$ indirectos a diversas actividades productivas. En Chile no existe una evaluación de la oferta de estos servicios, condición relevante para definir politicas y ordenamiento territorial. Este articulo presenta una metodologia para definir la localización espacial de los servicios ambientales de biodiversidad, captura de carbono, generación de agua de calidad y belleza escénica en la Región de los Rios, Chile.

Palabras Clave: Servicios ambientales, bosque nativo, análisis espacial

\section{A METHODOLOGICAL APPROACH FOR SPATIAL MODELING OF ECOSYSTEM SERVICES IN SOUTHERN CHILE}

\section{SUMMARY}

Forest environmental services have acquired a growing importance, generating direct and indirect benefits to several productive activities. In Chile does not exist an offer assessment of these services which is a relevant condition to define land policies. This article presents a methodology for defining spatial allocation of environmental services such us biodiversity, carbon sequestration, generation of water quality and scenic beauty in the Region de los Rios, Chile.

Key words: Environmental services, natural forests, spatial analysis

1-Ingeniero Forestal, Magister en Ciencias, Investigador, Instituto Forestal Sede Valdivia, Chile. gvergara@infor.cl 


\section{INTRODUCCIÓN}

Históricamente, el incremento no planificado en la producción de ciertos productos provenientes de los recursos naturales, tales como alimentos, madera, y materias primas, a menudo ha significado también una reducción del suministro de otros importantes elementos del ambiente, tales como la provisión de agua de calidad, el paisaje o la calidad del aire.

En este ámbito, nace hace ya más de un siglo la preocupación por las funciones y servicios generados por los ecosistemas, generalmente conocidos como servicios ambientales o ecosistémicos, los cuales son condiciones y procesos fundamentales para el funcionamiento general del ambiente, las actividades productivas y, más aún, para el bienestar general de las sociedades (MEA, 2003; Boyd y Banzhaf; 2006).

La relevancia del concepto de servicios ecosistémicos (SE) para el manejo de los ecosistemas reside en que es una ayuda para identificar el flujo de beneficios que surge de diferentes recursos naturales. Además, permite hacer un vinculo explicito entre el estado y funcionamiento de los ecosistemas y el bienestar humano, con lo cual puede constituir un medio para el desarrollo de politicas y la toma de decisiones (Balvanera y Cotler, 2007).

A pesar de la difusión que ha tenido el término en los últimos años, persisten aún diferencias de interpretación, que van desde un enfoque puramente funcional hasta otro más utilitario basado en la demanda. De esta manera y para tener un lenguaje común, este documento distingue los servicios, de las llamadas funciones y bienes ecosistémicos. Considerando la definición de Boyd y Banzhaf (2006) se tiene:

Funciones Ecosistémicas. Corresponden a las interacciones biológicas, quimicas y fisicas asociadas con los ecosistemas. Estas funciones son aquellas descritas por la biologia, las ciencias atmosféricas, la hidrologia etc. Las funciones son socialmente valiosas, pero no son servicios.

Activos Ecosistémicos. Son componentes intermedios de tipo fisico de la naturaleza. Estos bienes son intermedios, ya que ellos son necesarios para la producción de servicios, pero no son servicios en si mismos. Los bienes son los inputs para una función de producción ecológica que produce un servicio ecosistẻmico. En su nivel más alto estos activos pueden ser descritos como suelo, biota (vegetación, fauna y otros organismos), sistemas acuáticos (rios, lagos y humedales) y la atmósfera.

Estos activos naturales deben ser mantenidos en buenas condiciones por al menos dos razones. Primero, su vitalidad impacta directamente sobre su capacidad para continuar proveyendo inputs a la producción y, segundo, los activos naturales tienen valor en sí en la medida en que la gente derive valor por su existencia.

En la medida que los activos naturales son bien manejados, estos tienen la capacidad de proveer para las necesidades humanas en forma permanente. Esto contrasta con bienes construidos por el hombre los cuales tienen una vida útil limitada, como puede ser un auto o una industria. Los activos naturales pueden ser continuamente utilizados y 
regenerados luego por las funciones ecosistémicas, por lo cual el desafío es asegurar que estos activos no sean agotados antes de que puedan regenerarse.

Servicios Ecosistémicos. Son componentes de la naturaleza, directamente apreciados, consumidos o utilizados para producir bienestar humano. Estos pueden corresponder a servicios tangibles, tales como el agua limpia o el contenido de nitrógeno del suelo, o intangibles como la belleza escénica generada por bosques, montañas o cursos de agua.

Existe conciencia que la conservación y mantenimiento de las funciones ecosistémicas asociadas al espacio rural demandan el uso y manejo más responsable de los recursos naturales, pero siendo diversos los actores en tamaño, objetivos y escalas de trabajo, se requieren reglas claras y acciones coordinadas con enfoque espacial.

La medición de estos componentes contiene una alta dependencia de escala en términos de tiempo y espacio. Esto debido a que el valor de los servicios ecosistémicos es altamente dependiente de su localización en el paisaje, de la escala sobre la cual los servicios son provistos y del tiempo en el cual estos son proporcionados (Boyd, 2004; Boyd y Wainger, 2003).

La búsqueda de indicadores de oferta de servicios ambientales en Chile ayudará al conocimiento de dónde existe mayor potencial de desarrollo de servicios ecosistémicos en el pais y en qué cantidad, sirviendo de información base para jerarquizar zonas donde futuros instrumentos económicos puedan operar como incentivos a un manejo sustentable de los recursos. Por ello, entre los beneficios de este tipo de iniciativas se destacan el aporte a la conservación del bosque nativo, a través del fomento a actividades productivas compatibles con este objetivo, y el aporte al desarrollo local y regional, a través del impulso a actividades económicas en expansión tales como turismo, pesca deportiva y producción de salmónidos, cuyo desarrollo depende fuertemente de la calidad de los servicios ecosistémicos asociados.

\section{OBJETIVOS}

\section{Objetivo General}

El objetivo del estudio es dar una mirada hacia la integridad de los ecosistemas y los servicios que son provistos actualmente, para establecer las bases para una evaluación más detallada de lo que podria ocurrir con estos bajo un conjunto de escenarios en el futuro.

\section{Objetivos Especificos}

Identificar indicadores que definan la integridad de los ecosistemas y orienten el establecimiento de esquemas de pago por servicios ecosistémicos.

Identificar los servicios ecosistémicos de alta prioridad para estudios en profundidad y propuestas de manejo.

Identificar y promover unidades de conteo ambiental. 


\section{MATERIAL Y MÉTODO}

\section{Caracterización del Área de Análisis}

El área de análisis corresponde a la Región de Los Rios, la cual presenta una superficie aproximada de $18.429 \mathrm{~km}^{2}$ distribuida en 2 provincias y 12 comunas. Su población estimada es de 356.396 habitantes, según el Censo 2002 (INE, 2004).

La población rural de la Provincia de Valdivia corresponde a un $26 \%$ de la población total y su densidad es de $25 \mathrm{hab} / \mathrm{km}^{2}$, lo cual está fuertemente influenciado por la presencia de la capital regional. En tanto la Provincia del Ranco presenta un $47 \%$ de su población en áreas rurales y solo $12 \mathrm{hab} / \mathrm{km}^{2}$, lo que indica una alta dependencia de la población de sus recursos.

Otro aspecto relevante está dado por los niveles de escolaridad, analfabetismo, indigencia y pobreza de la Región, los cuales son particularmente elevados en comunas andinas, tales como Lago Ranco y Futrono y, en comunas de la depresión intermedia, como Los Lagos, Lanco y Máfil.

\section{Cuadro $\mathrm{N}^{\circ} 1$ \\ USO DEL SUELO A NIVEL REGIONAL}

\begin{tabular}{|c|c|c|}
\hline Uso actual & $\begin{array}{c}\text { Superficie Regional } \\
\text { (M ha) }\end{array}$ & $\begin{array}{l}\text { Proporción } \\
(\%)\end{array}$ \\
\hline Terrenos Agricolas & 14 & 0,7 \\
\hline Praderas y Matorrales & 589 & 31,9 \\
\hline Bosques & 1.032 & 56,0 \\
\hline Terrenos Improductivos & 87 & 4.7 \\
\hline Aguas Continentales & 108 & 5,8 \\
\hline Áreas No Reconocidas & 9 & 0,5 \\
\hline Total & 1.841 & 100,0 \\
\hline
\end{tabular}

(Fuente: CONAF-CONAMA-BIRF, 1994-1997) 


\section{Cuadro $\mathrm{N}^{\circ} 2$ \\ DISTRIBUCIÓN DE LOS BOSQUES A NIVEL REGIONAL}

\begin{tabular}{|c|c|c|}
\hline Uso & $\begin{array}{c}\text { Superficie Regional } \\
\text { (M ha) }\end{array}$ & $\begin{array}{l}\text { Proporción } \\
(\%)\end{array}$ \\
\hline PLANTACIONES & 180.349 & 9,8 \\
\hline BOSQUE NATIVO & 851.651 & 46,2 \\
\hline Bosque Adulto & 466.604 & 25,3 \\
\hline Renoval & 245.706 & 13,3 \\
\hline Bosque Adulto-Renoval & 80.109 & 4,3 \\
\hline Bosque Achaparrado & 59.232 & 3,2 \\
\hline
\end{tabular}

(Fuente: CONAF-CONAMA-BIRF, 1994-1997)

\section{Funciones y Servicios Considerados}

Las funciones y servicios bajo análisis en este estudio son los siguientes:

Regulación del clima. Referente a las funciones provistas por la vegetación y que regulan la composición atmosférica y los patrones del clima, creando un ambiente habitable. Algunos indicadores asociados corresponde al stock de carbono, las tasas de crecimiento entre otros.

Biodiversidad. Determina el rol de la biodiversidad como un componente fundamental para el desarrollo sustentable de actividades productivas y la generación de funciones y servicios en el mediano y largo plazo. Ej. Polinización de cultivos y vegetación natural, control de plagas.

Producción y regulación hidrica. Definido por las diferentes funciones y servicios relacionados con la producción (cantidad y calidad) de agua, asi como la mantención de la sanidad el suelo, la regulación de flujos de cursos de agua y los niveles de aguas subterráneas.

Belleza escénica. Provisión de beneficios generados por el paisaje mediante componentes particulares del medio, tales bosques, relieve, fauna y otros, los cuales producen condiciones de gran valor visual.

\section{Marco Metodológico General}

Bajo una perspectiva de enfoque transdisciplinario, el Instituto Forestal (INFOR) buscó la conformación de alianzas y convenios con otras instituciones especializadas en el manejo y administración de recursos naturales. De esta modo, conformó un análisis multifuente, de manera de analizar los aspectos ecológicos, espaciales y socioeconómicos que se derivan de las funciones y servicios ecosistémicos en distintos puntos del territorio. 
El enfoque basal de este estudio considera la utilización de indicadores e indices agregados de provisión para las diferentes funciones y servicios ya descritos. En forma adicional, se deriva un conjunto de indicadores de beneficios relacionados a la existencia de estos componentes, considerando las condiciones ecológicas y sociales de la Región.

Las razones de utilizar un sistema de indicadores en este estudio son las siguientes:

Los indicadores pueden ser cuidadosamente estructurados para ayudar a los tomadores de decisión a organizar sus prioridades en torno a la valoración, comunicar los beneficios de los SE y apoyar el análisis regional y la comunicación de la interdependencia espacial a diferentes grupos de interés.

Los indicadores son menos costosos de generar, permitiendo la evaluación del paisaje de múltiples servicios a grandes escalas.

Permiten catastrar y comparar situaciones medioambientales de unidades territoriales de forma integral.

Pueden constituir "proxies" que indiquen la existencia o la generación de un servicio. simplificando la complejidad de los ecosistemas.

Bajo estas consideraciones, la estructura metodológica general del estudio contempla las etapas identificadas en la Figura $\mathrm{N}^{\circ} 1$.

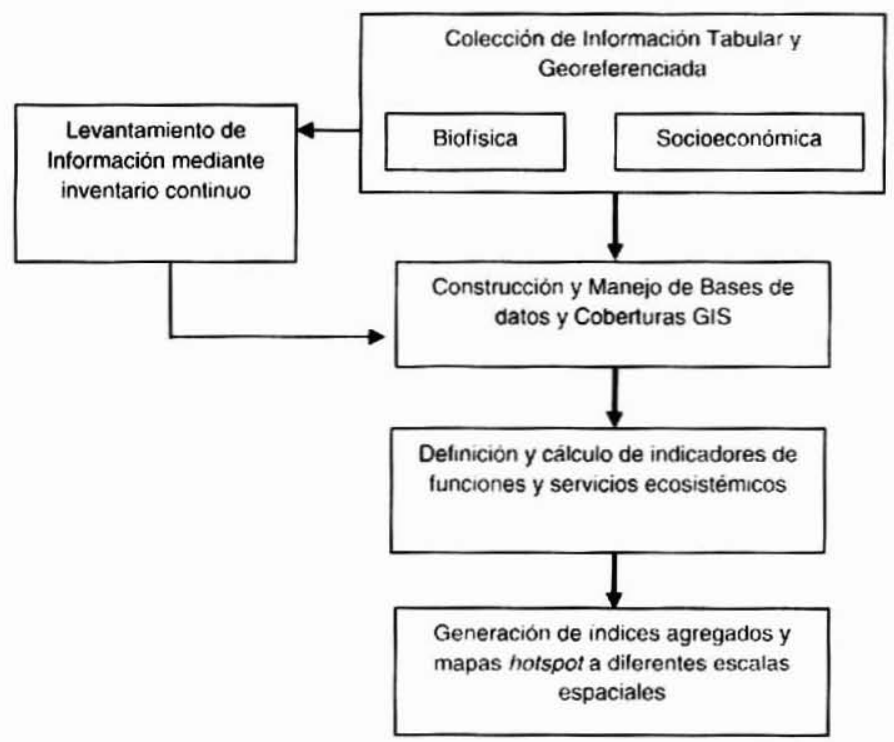

Figura $N^{\circ} 1$

ESQUEMA METODOLÓGICO UTILIZADO EN EL ESTUDIO 


\title{
Colección de Información Tabular y Georeferenciada
}

En la etapa inicial de este trabajo, se recolectó información secundaria en forma de tablas, las cuales fueron transformadas en forma georeferenciada digital. De igual forma se obtuvo información digital ya procesada, la cual fue facilitada por diversas instituciones nacionales.

El modelo digital de elevación (DEM) fue obtenido de la página de la NASA en EE.UU. y dispone de una resolución espacial de $50 \mathrm{~m}$.

Para realizar esta tarea, el inventario se ejecutó sobre la bases de la siguiente información:

\author{
Geoinformación digital disponible \\ Sensores remotos \\ Inventario de bosque nativo \\ Información económica \\ Censo población \\ Censo agropecuario \\ Toma de datos
}

La información final se presenta en forma tabular y cartográfica para distintas áreas y escalas, de modo que pueda constituir una nueva forma de visualizar el territorio y sus formas de producción.

\section{Construcción, Manejo de Bases de datos y Coberturas GIS}

El análisis y homogenización de la información fue realizado con los sofwares Arcview 3.2 y ArcGIS 9.3. Las bases de datos de diferentes coberturas (shapes) fueron revisadas y corregidas con información auxiliar disponible. La información GIS se integró en un proyecto único (formato $\mathrm{mxd}$ ) de ArcGIS para facilitar su análisis y el despliegue de información.

\section{Definición y Cálculo de Indicadores de Funciones y Servicios Ecosistémicos}

Para el análisis de los cuatro servicios ecosistémicos considerados se tuvo como marco los siguientes elementos:

\section{Biodiversidad}

La diversidad biológica significa la variabilidad entre organismos vivientes de todas las fuentes, incluye la diversidad dentro de las especies, entre especies y de ecosistemas 
(CBD, 1992). Es un concepto amplio y de gran complejidad, principalmente en lo que respecta a su cuantificación.

La diversidad biológica fue analizada en tres componentes primarios importantes: La composición, la estructura y la función. En este caso se han desarrollado indicadores para los dos primeros componentes.

\section{Carbono}

Para el análisis del Carbono, se consideran dos temas importantes, el primero los terrenos que pueden participar en proyectos REDD (UN-REDD Programme Fund. United Nations Collaborative Programme on Reducing Emissions from Deforestation and Forest Degradation in Developing Countries), que permitirian el ingreso al mercado del carbono y, el segundo, la contabilización del stock de carbono existente en los bosques en pie que actualmente existen en la Región.

Para la determinación del stock de carbono de los ecosistemas forestales, es necesario cuantificar la biomasa de éstos, para finalmente determinar el carbono existente, ya que el carbono se acumula en la biomasa del ecosistema forestal y la biomasa es definida como el peso, o estimación equivalente, de materia orgánica que existe en un determinado ecosistema forestal.

El primer paso fue determinar los ecosistemas forestales existentes en la Región, lo cual se realiza de acuerdo a la clasificación de usos de la tierra elaborada por el Catastro y Evaluación del Recurso Vegetacional Nativo del pais. Se presentan los niveles de biomasa y contenido de carbono sobre el suelo a nivel regional. La biomasa se calculó a partir del volumen bruto fustal y después se expandió para considerar toda la biomasa aérea. Finalmente y como tercer paso, después de determinar la biomasa de los árboles vivos y la biomasa de árboles muertos y residuos gruesos, se calcula la cantidad de carbono. Para esto se utiliza como base el trabajo realizado por el proyecto FONDEF D98|1076, el cual determinó el contenido de carbono considerando especies del tipo forestal Siempreverde y Roble-Rauli-Coigüe, se utiliza el valor promedio de contenido total de carbono que fue $49,64 \%$ (Gayoso y Guerra, 2005).

\section{Belleza Escénica (Paisaje)}

Los paisajes constituyen un componente integral de los recursos forestales nativos. La evaluación del impacto visual debiera ser parte del plan de desarrollo forestal y del plan de manejo, esto considerando principalmente que, la Región recibe año a año, cientos de turistas que buscan lugares de esparcimiento con espacios naturales o autóctonos. Para el análisis del paisaje, se utilizó los elementos considerados por el Código de Prácticas Forestales de British Columbia, Canadá (2001). Dicho proceso se divide en seis fases:

El inventario del paisaje visual

El análisis del paisaje visual 


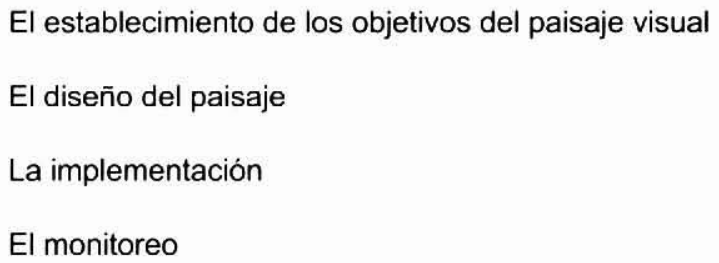

En el caso del inventario de recursos visuales presentes en cada área de estudio, se consideraron los siguientes elementos:

\author{
Puntos de interés escénico \\ Marcas visuales \\ Alteraciones mayores \\ Cursos y cuerpos de agua \\ Cubiertas vegetales dominantes \\ Áreas singulares
}

A esto se agrega el concepto de accesibilidad, elemento que determina la funcionalidad de un determinado recurso visual.

\title{
Agua
}

La unidad natural para organizar adecuadamente las actividades de manejo y controlar los efectos sobre el ambiente es la cuenca. Esta corresponde a un área delineada topográficamente que es drenada por un sistema de cauces. Para propósitos de planificación y gestión, esta es considerada como una unidad de respuesta hidrológica, una unidad físico-biológica y una unidad socioeconómica (Folliot, 1997). De esta manera, se evaluó la generación de agua de calidad considerando el nivel de cobertura boscosa y el uso del suelo, las zonas de protección de cauces y la pendiente del terreno.

\section{Generación de Índices Agregados y Mapas Hotspot a Diferentes Escalas Espaciales}

Los indicadores agregados a nivel espacial fueron derivados mediante geoprocesamiento en ArcGls 9.3. Diferentes procesos tales como zonas de influencia, disolución, agregación, proximidad y otros, permitieron construir diferentes mapas temáticos agregados a nivel regional.

La construcción de estos mapas tiene por objetivo ayudar a los tomadores de decisiones a orientar la definición de programas, tomar medidas de protección o facilitar posibles inversiones. Se espera además que pueda promover la conciencia pública acerca del potencial ecológico y 
económico que presentan los bosques del pais. Finalmente, pretende ser una herramienta de apoyo para orientar esquemas de compensación por servicios ecosistémicos y la consecuente protección de este capital natural.

\section{RESULTADOS}

Considerando la experiencia internacional, los resultados de este proyecto se han enmarcado en cuatro de los servicios ecosistémicos de los bosques, con mayor posibilidad de mercado. Estos corresponden a la captura de carbono, generación de agua limpia, belleza escénica y biodiversidad. Los resultados se presentan en función de los mapas generados.

\section{Biodiversidad}

Los tipos forestales son un indicador simple de la biodiversidad de los bosques por cuando indican su composición o la asociación de especies que crecen en el bosque ed acuerdo a cada uno (Figura $\mathrm{N}^{\circ} 2$ ).

Entre los tipos forestales más notables en la Región están los Tipos Forestales Araucaria y Alerce, los cuales son definidos por la presencia de estas singulares especies. El Tipo Forestal Araucaria está presente en la zona nororiental de la Cordillera de los Andes, correspondiente al sector norte de la Comuna de Panguipulli. Ésta zona está siendo considerada para anexarla al Parque Nacional Villarrica debido a su importancia ecológica y paisajística.

El Tipo Forestal Alerce se encuentra sobre la Cordillera de la Costa, al sur de Corral, y es parte hoy del Monumento Nacional Alerce Costero. Se ha anexado además a esta zona de protección, la Reserva Costera Valdiviana, una reserva privada que busca además la conservación del bosque simpreverde amenazado fuertemente por explotaciones madereras en las décadas anteriores. 


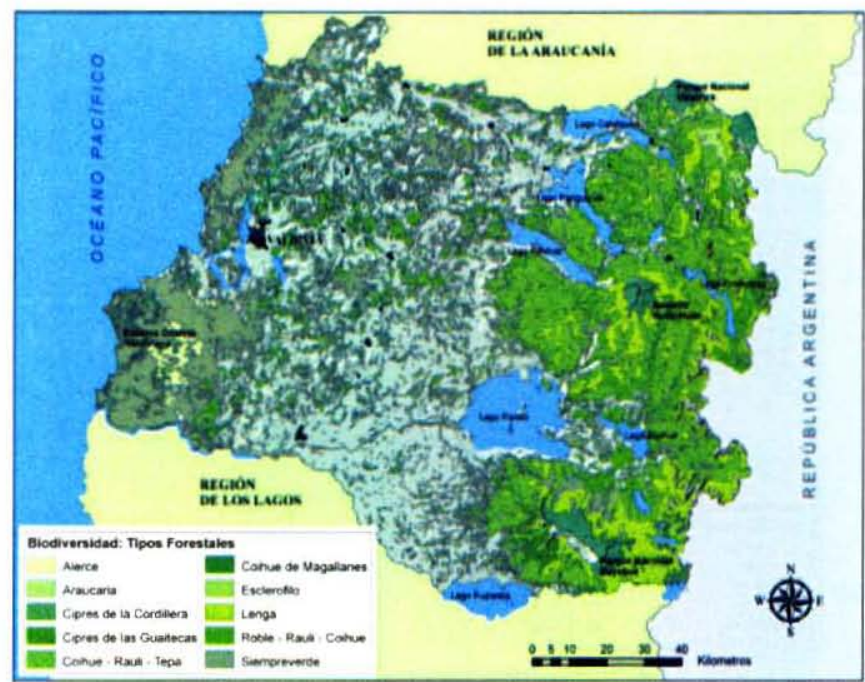

Figura $\mathrm{N}^{\circ} 2$

INDICADOR DE BIODIVERSIDAD

TIPOS FORESTALES DEL BOSQUE NATIVO CHILENO EN LA REGIÓN DE LOS RIOS

El bosque siempreverde es uno de los tipos forestales más diversos tanto floristicamente como estructuralmente, y se encuentra en las Cordilleras de los Andes y de la Costa, principalmente en zonas de mayores pendientes o sin acceso de caminos. Junto a los tipos forestales Coihue - Rauli - Tepa y Lenga constituyen la mayor masa forestal en la Región.

La depresión intermedia es dominada por el tipo forestal Roble - Rauli - Coihue, en muchas de las áreas con suelos más productivos y de menores pendientes, razón por la cual, estos bosques han sido altamente degradados y fragmentados para habilitar zonas para cultivos agricolas o cria de ganado.

La Figura $\mathrm{N}^{\circ} 3$ muestra el indice de diversidad de parches (IDP), el cual es un indicador del nivel de fragmentación de bosques a nivel de paisaje. De acuerdo a este indicador, los bosques mejor conservados de la Región, se encuentran en la Cordillera de los Andes, los cuales han permanecido en el área, debido a los altos costos de construcción de caminos y a la baja en los precios de la madera, factores que han ayudado a su conservación hasta la fecha.

El área sur de la Cordillera de la Costa (Comunas de Corral y La Unión), también presenta menores indices de fragmentación, lo cual probablemente se mantendrá debido a su estatus de zona de conservación. Sin embargo, la zona de la depresión intermedia muestra una fuerte presión al cambio de uso, lo cual ha derivado en una alta fragmentación de sus bosques. 


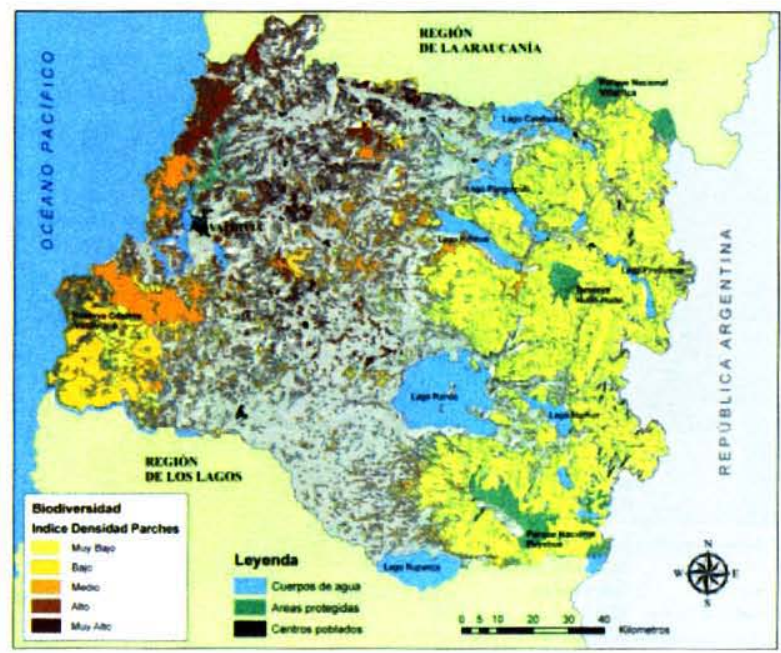

Figura $\mathrm{N}^{\circ} 3$

\section{INDICE DE DENSIDAD DE PARCHES (NIVEL GENERAL) PARA LA REGIÓN DE LOS Ríos}

A nivel comunal, Lanco y Paillaco presentan los mayores indices de fragmentación de sus bosques, situación coincidente con la actividad principal (agroganadera) que se desarrolla en ambas comunas (Figura $\mathrm{N}^{\circ} 4$ ). En una condición opuesta están las Comunas de Futrono, Lago Ranco y Panguipulli, las cuales concentran su territorio en la Cordillera de los Andes

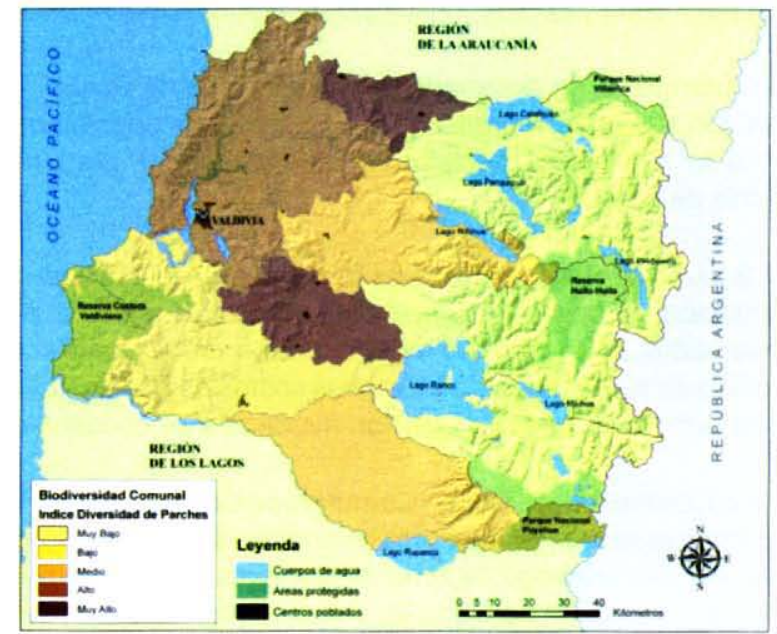

Figura $\mathrm{N}^{\circ} 4$

ÍNDICE DE DENSIDAD DE PARCHES A NIVEL COMUNAL 
Los tipos forestales con mayores indices de fragmentación corresponden a Roble-RauliCoihue y Siempreverde. En el caso de este último, la mayoria de los bosques en la Cordillera de la Costa al norte y sur de Valdivia (principalmente en la vertiente oriental) presentan fuertes presiones ambientales para utilización de leña para calefacción (Figura $\mathrm{N}^{\circ} 5$ ).

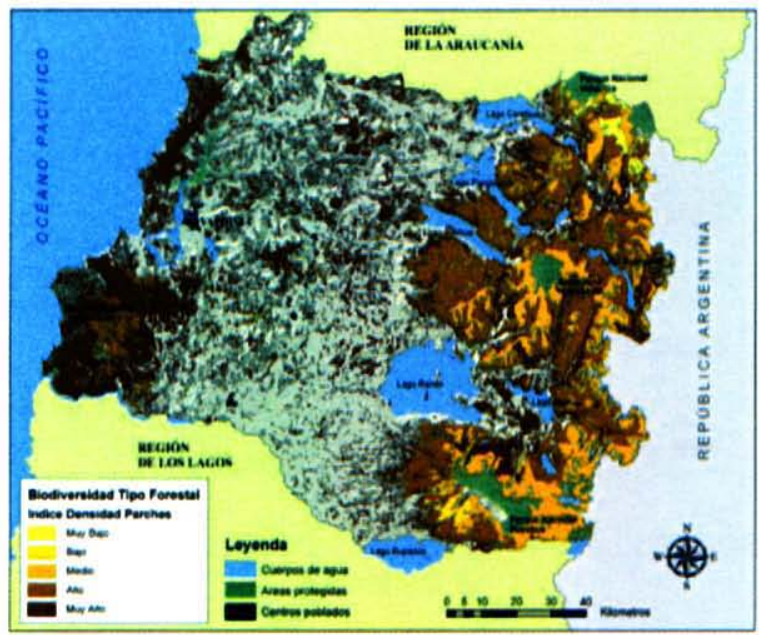

Figura $\mathbf{N}^{\circ} 5$

INDICE DE DENSIDAD DE PARCHES A NIVEL DE TIPO FORESTAL

\section{Carbono}

La función de captura de carbono por parte de los bosques fue definida por la biomasa viva sobre el suelo, correspondiente a todo el material leñoso creciendo sobre la superficie o piso del bosque. Esta función ecosistémica del bosque es bien representada por el volumen de los árboles en pie, tradicionalmente muestreada en los inventarios forestales.

La Figura $\mathrm{N}^{\circ} 6$ muestra un mapa sinóptico del volumen de biomasa sobre el suelo en la Región de los Ríos. De forma coincidente con la información anterior, los mayores niveles de biomasa están concentrados en los tipos forestales que crecen en ambas cordilleras, valores que varían ampliamente debido a la variabilidad intrinseca de los ecosistemas, así como sor intervenciones antrópicas sobre estos bosques. 


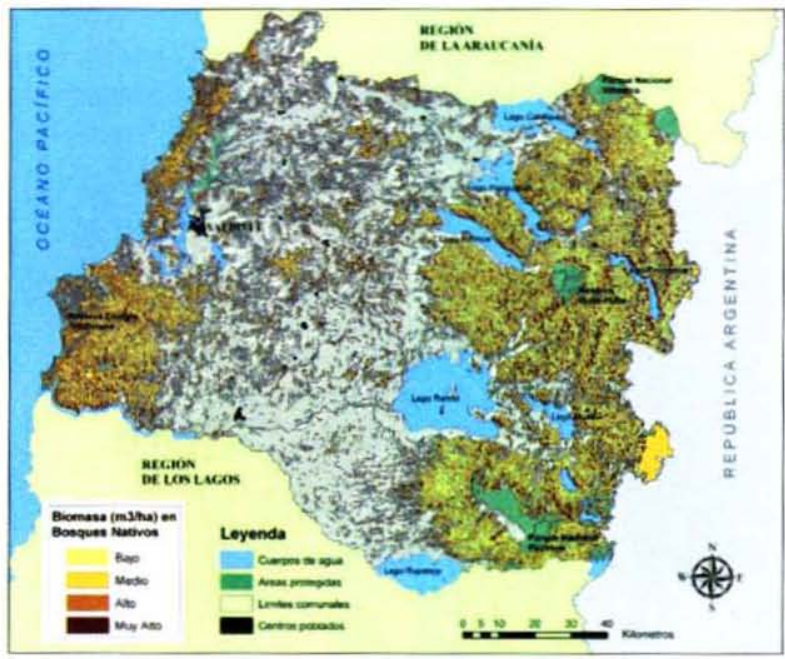

Figura $\mathrm{N}^{\circ} 6$

BIOMASA VIVA SOBRE EL SUELO EN LOS TIPOS FORESTALES DE LA REGIÓN DE LOS RíOS.

El carbono efectivamente retenido en la estructura de los árboles está en función de la densidad y caracteristicas de las estructuras leñosas de las diversas especies. Mayores posibilidades de realización de proyectos de Reducción de Emisiones por Deforestación y Degradación de Bosques (REDD) son probables en ambas cordilleras (Figura $\mathrm{N}^{\circ} 7$ ).

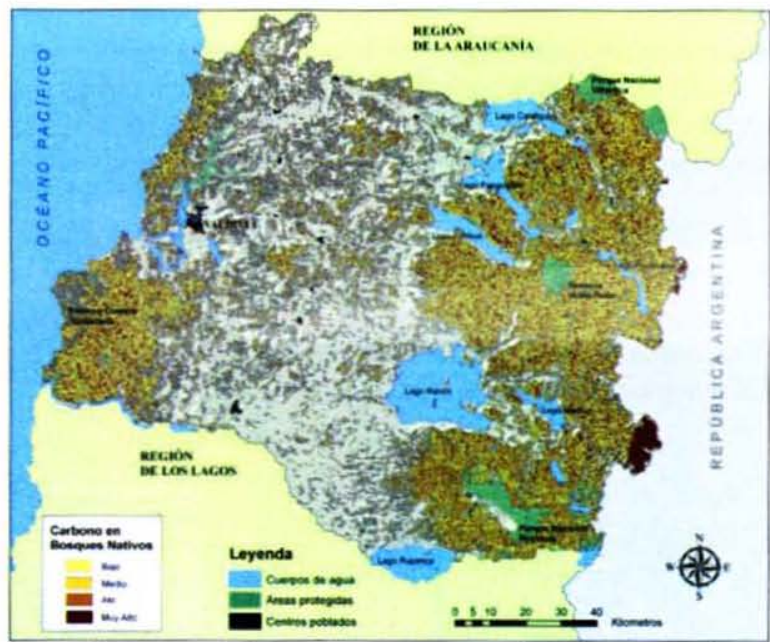

Figura $\mathrm{N}^{\circ} 7$

SUMIDEROS DE CARBONO FORESTAL EN LA REGIÓN DE LOS RIOS 


\section{Agua}

Los grandes sistemas hidrológicos en la Región están definidos por la presencia de los Rios Bueno, San Pedro y Cruces. Dichos sistemas fueron separados en 16 subcuencas con el fin de analizar distintas posiciones fisiográficas del territorio (Figura $\mathrm{N}^{\circ} 8$ ). Considerando el nivel de protección y uso que se le da a las riveras de los cauces en cada subcuenca, se tiene que aquellas de la región andina, tales como Maihue, Pirehueico y Neltume y Corral en la zona costera, son los sistemas hidrológicos que potencialmente pueden producir agua de mejor calidad.

Una condición totalmente distinta se aprecia en la cuenca denominada "Bueno", que es la que presenta el menor potencial y donde se desarrolla la mayor actividad agroganadera de la Región.

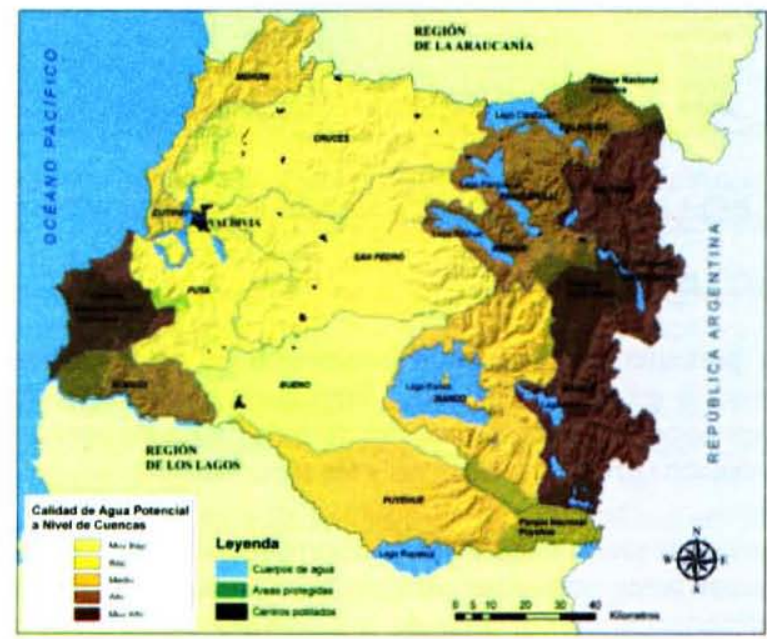

Figura 8

CALIDAD DE AGUA POTENCIAL A NIVEL DE CUENCAS EN LA REGIÓN DE LOS RÍOS.

\section{Paisaje}

La calidad del paisaje o belleza escénica de la Región fue derivada como una función de las áreas de interés en el paisaje (lagos, rios, termas, bosques etc), su ubicación geográfica dentro de la cuenca visual y la accesibilidad a la zona.

Las áreas de mayor potencial en este sentido corresponden a la zona costera valdiviana, la cuenca de los Lagos Ranco y Maihue, y el área comprendida entre los Lagos Calafquén. Panguipulli y Riñihue en la Cordillera de los Andes. Muchas de estas áreas tienen un gran potencial, que mejoraria si existiera una mayor infraestructura que permitiera el acceso a estas zonas de la Región. 


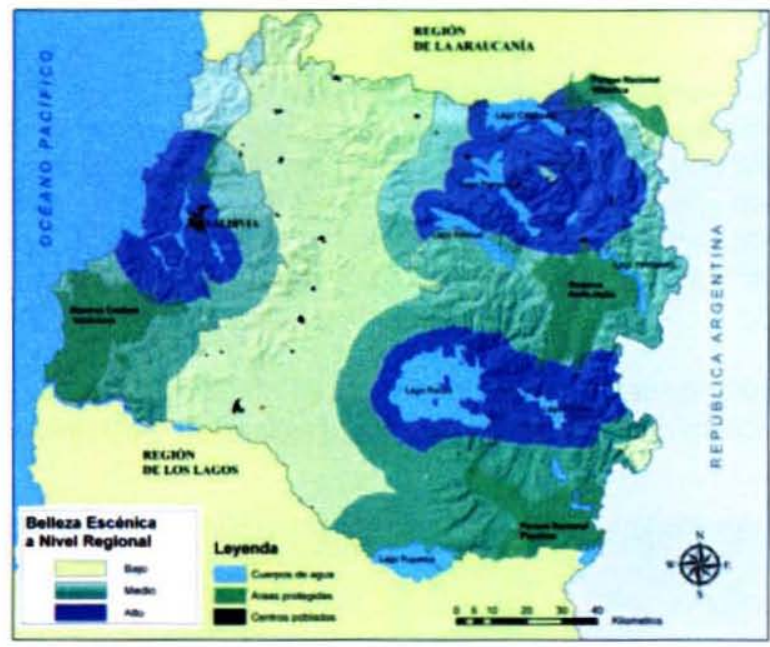

Figura $\mathbf{N}^{\circ} 9$

NIVELES DE BELLEZA ESCÉNICA O PAISAJE EN LA REGIÓN DE LOS RIOS

\section{DISCUSIÓN Y CONCLUSIONES}

Este articulo presenta los elementos fundamentales de la realización del inventario y mapeo de los servicios ecosistémicos en la Región de los Rios. Se han incorporado los enfoques a nivel internacional, de modo de determinar la oferta de servicios que se producen en el territorio y su relación con las condiciones y las tendencias de los ecosistemas.

De los antecedentes revisados y la información generada en este estudio, se desprende que existen al menos tres áreas relevantes para promover sistemas de compensación ambiental en la Región. Estos corresponden al área de la Cordillera de la Costa al sur de Corral, la cuenca de los Lagos Maihue y Ranco, y el sector comprendido entre los lagos Calafquén y Riñihue. Debido a la gran riqueza en recursos naturales, la geografia de la zona y los servicios ecosistémicos que estas áreas poseen, existe mayor probabilidad de desarrollar sistemas de compensación que promuevan el uso sustentable de los recursos de esta Región.

Se espera además, que la generación de esta información de servicios ecosistémicos pueda constituir una herramienta de planificación regional y que permita la identificación de potencialidades y falencias en la provisión de servicios relevantes de la economia local. Mediante este proceso se espera aportar a que privados e instituciones públicas puedan aplicar y promover regulaciones y esquemas de manejo de los recursos que fortalezcan la generación sustentable de estos servicios. 


\section{REFERENCIAS}

Balvanera, P.; J. Cotler. 2007. Gaceta Gaceta ecológica número especial 84-85: 8-15. Instituto Nacional de Ecologia, México

Banzhaf, S. and Boyd, J., 2004. Indexes and Indicators of Ecosystem Services. Resources for the Future. Washington, D.C. $30 \mathrm{p}$.

Boyd, J., 2004. What's nature worth? Using Indicators to Open the Black Box of Ecologicel Valuation. Resources for the Future.5p.

Boyd, J. and Banzhaf, S., 2006. What are ecosystem services? The need for standardiz त environmental accounting units. Resources for the future. Wahington DC. 26 p.

Boyd, J. and Wainger, L., 2003. Measuring Ecosystem Service Benefits: The Use of Lands Analysis to Evaluate Environmental Trades and Compensation. Discussion Paper 02-tu Resources for the future. Washington, D.C. $156 \mathrm{p}$.

CONAF-CONAMA-BIRF, 1994-1997. Catastro y Evaluación de los Recursos Vegetacionales Nativos de Chile". 1994-1997. Incluye corrección por monitoreo y actualización, 1998.

Folliot, P. (1997). Watershed management in the southwest: Training course in watershed management, University of Arizona. 12 p.

Gayoso J, J Guerra 2005. Contenido de carbono en la biomasa aérea de bosques nativos de Chile. Bosque 26 (2):33-38 Government of British Columbia, Canadá. 2001. Visual Impact Assessment Guidebook.

In: http://www.for.gov.bc.ca/TASB/LEGSREGS/FPC/FPCGUIDE/visual/Httoc.htm Revisada en octubre 2009.

INSTITUTO NACIONAL DE ESTADISTICAS, 2004. Anuario de turismo 2003. Santiago- Chile. $95 \mathrm{p}$.

Millenium Ecosystem Assessment, 2003. Ecosystems and human well-being: a framework for assessment. Washington DC. $266 \mathrm{p}$.

NACIONES UNIDAS, 1992. Convenio sobre la diversidad biológica. Rio de Janeiro. 42 p. 
